\title{
Manager der Versorgung - auch im SGB V längst Pflicht
}

Jeder Patient hat ein Recht auf eine gute Koordination seiner Behandlung über die Grenzen von Sektoren und Versicherungen hinweg. So steht es in den Sozialgesetzbüchern, allen voran dem SGBV. Doch die meisten Ärzte und Patienten wissen nichts davon. Und manch Krankenhaus löst seine Verpflichtung dazu erst gar nicht ein.

Jeder hat schon mal von dem Zirkus gehört, der losgeht, wenn eine Reha nicht bewilligt wird, oder dringend ein Pflegedienst her muss, von dem man nicht weiß, was die Kasse nun bezahlt oder auch nicht. Anders formuliert: „Man müsste nur einen Ansprechpartner haben ..."

Diesen Stoßseufzer dürften viele Patienten schon mal getan haben, wenn sie an den Brüchen im stark sektorierten hiesigen Gesundheitswesen nicht weiter wussten. Das Zitat stammt zugleich aus dem Titel einer Dissertation [1], die sich damit beschäftigt, „wie major-beinamputierte Menschen ihre rehabilitative Versorgung erleben“. Verfasst von Ulrike Michaelis, wissenschaftliche Mitarbeiterin an der Hochschule für Angewandte Wissenschaften Hamburg - basierend auf Einzelinterviews von 28 Menschen in den Jahren 2009 und 2010, die zu dem Zeitpunkt jeweils bereits ein halbes Jahr aus dem Krankenhaus nach der Amputation entlassen waren. Solch eine Amputation ist ein Schicksal, das jährlich gut 20000 Menschen hierzulande erleiden, Hauptursachen sind Gefäßverschlüsse und eine Diabeteserkrankung.

Michaelis wollte wissen, wie gut die Betroffenen im Alltag wieder zurechtkommen, welchen Unterstützungsbedarf sie sehen, welche Hilfe sie sich wünschen und welche sie erhalten.

Fazit: Es dominiert ein Gefühl des Alleingelassenseins, viele beklagten, sich alle Infos selber besorgen zu müssen, zu wenig Hilfe im Dschungel von verschiedenen Versicherungsträgern und potenziellen Versorgern zu bekommen. Viele Teilnehmer sehen ein „Zergliedern von Zustän- digkeiten“ als Hauptproblem, die Hilfe zu organisieren, die sie benötigen.

„Also ich denke mal, es wäre optimal, wenn es einen, ich sage mal, wenn es einen Reha-Berater geben würde, ..., der einen auch richtig aufklärt", meint zum Beispiel Herr T., denn das Gesundheitssystem sei ein völlig „unkontrolliertes Selbstbedienungssystem“. Es hinge schon vom Glück ab, ob man nun an jemanden gerate oder nicht.

Frau S. vermisst: „... dass überhaupt mal einer hilft, dass man eine Wohnung findet oder sagt, wo ein Arzt ist, wo man hingehen kann im Rollstuhl und so, da ist nichts." Eineinhalb Jahre, moniert ein anderer, habe es gedauert, bis ein Rollstuhl endlich von der Kasse kam. Auch die „Sache mit dem Behindertenausweis" habe man sich mühsam selbst erarbeiten müssen. Man kriege das Gefühl, bilanziert wieder ein anderer Betroffener düster, dass man eigentlich nichts mehr wert sei.

Es sei ein typischer Querschnitt durch die Erfahrungs- und Lebenswelt solcher Patienten - erklärt Michaelis. Denn obendrein hat eine 2. Studie an dann 515 Betroffenen das Ergebnis statistisch auch quantifizierbar reproduziert [2]. „Wir sehen, dass sehr viele Betroffene diese Probleme haben“, erklärt Michaelis.

Es gab auch eine Konsequenz aus der Forschung - sie lief in einem BMBF-geförderten Projekt zur Förderung der Selbstpflegekompetenzen von Menschen mit Amputationen („SeKom“ [3]). Die Pflegewissenschaftlerin hat an einem Curriculum für eine Schulung zur Care ManagerIn Amputationsbegleitung mitgearbeitet [4]. Gedacht ist der 2-wöchige Kurs zur Weiterbildung primär von Pflegekräften und Physiotherapeuten. Allerdings lief der Kurs bislang nur einmal im Rahmen des Projekts.

Ideal, so Michealis, sei, wenn Kassen ein Modellprojekt aufsetzen könnten, wo solch geschulte Kräfte hinterher zum Einsatz kämen. „Wir würden dabei auch prüfen wollen, welche Kosten solch eine bes- sere Versorgung für die Patienten macht und welche Einsparungen es andererseits durch gezielte Versorgung gibt“, betont Michaelis. Doch die bislang angefragten Kassen haben abgelehnt. Michaelis: „Es ist uns leider nicht gelungen, Kassen zu überzeugen.“

Man darf hinzufügen: Streng genommen braucht es für solche Hilfe keine Modellprojekte. Denn eigentlich, ja eigentlich zieht sich durch alle Sozialgesetzbücher längst die Vorschrift, dass Patienten ein Recht auf Unterstützung haben, nach medizinischer Versorgung auch im Alltag bestmöglich wieder Tritt zu fassen. Und ein gut Teil dieser Unterstützung besteht in der aktiven Information der Betroffenen - und sei es darüber, wo sie welchen Antrag wie stellen sollten und können.

Paragrafen gefällig? Wie wäre es mit $\S 11$, Absatz 4 des SGBV: „Versicherte haben Anspruch auf ein Versorgungsmanagement insbesondere zur Lösung von Problemen beim Übergang in die verschiedenen Versorgungsbereiche; dies umfasst auch die fachärztliche Anschlussversorgung. Die betroffenen Leistungserbringer sorgen für eine sachgerechte Anschlussversorgung des Versicherten und übermitteln sich gegenseitig die erforderlichen Informationen. Sie sind zur Erfüllung dieser Aufgabe von den Krankenkassen zu unterstützen. In das Versorgungsmanagement sind die Pflegeeinrichtungen einzubeziehen; dabei ist eine enge Zusammenarbeit mit Pflegeberatern und Pflegeberaterinnen nach §7a des Elften Buches zu gewährleisten..."

Reicht nicht? Dann nehmen Sie §39 Abs. 1a: „Die Krankenhausbehandlung umfasst ein Entlassmanagement zur Unterstützung einer sektorenübergreifenden Versorgung der Versicherten beim Übergang in die Versorgung nach Krankenhausbehandlung [...] Der Versicherte hat gegenüber der Krankenkasse einen Anspruch auf Unterstützung des Entlassmanagements nach Satz 1 [...] soweit Hilfen durch die Pflegeversicherung in Be- 
tracht kommen, kooperieren Krankenund Pflegekassen miteinander.“

Solch ein Entlassungsmanagement umzusetzen, ist seit Anfang 2016 für alle Kliniken verpflichtend - durch das 2015 neu gegebene Krankenhausstrukturgesetz.

Immerhin hatte der Gemeinsame Bundesausschuss G-BA Ende 2015 bereits festgezurrt, dass auch Klinikärzte jetzt in diesem Rahmen für die 1. Woche daheim Rezepte für Arzneimittel, häusliche Krankenpflege, Heil- und Hilfsmittel ausstellen dürfen [5].

Vor Ort aber wird der Patient davon oft nicht sonderlich viel wahrnehmen. Ein eigentlich vorgesehener Rahmenplan nach diesem Paragrafen lässt auf sich warten. Denn die „weiteren Einzelheiten“ sollten der Spitzenverband Bund der Krankenkassen, auch als Spitzenverband Bund der Pflegekassen, die Kassenärztliche Bundesvereinigung und die Deutsche Krankenhausgesellschaft unter Berücksichtigung der Richtlinien des Gemeinsamen Bundesausschusses bis zum 31. Dezember 2015 in einem Rahmenvertrag regeln.

Und auch wenn der Vertrag noch kommen sollte, bleibt zu vermuten, dass es sehr vom einzelnen Krankenhaus abhängt, was es als Management für den Tag der Entlassung bietet. „Wo kein Kläger, da kein Richter“, weiß Professor Dr. Peter Löcherbach von der Katholischen Hochschule Mainz. Das Sozialgesetzbuch sei voll von Beziehungsregeln - Stellen, an denen der Gesetzgeber die Leistungsträger und die Leistungserbringer von Krankenhaus bis ambulantem Pflegedienst, von der RehaKlinik bis zum Sozialamt verpflichtet, den Patienten in den Mittelpunkt zu stellen und sich für sein Wohl abzusprechen und zu koordinieren. Löcherbach: „Allein, es gibt keine Sanktionen für den Fall, dass eben nichts passiert - wo wollen Sie da Ihr Recht einfordern.“

Als Vorstandsvorsitzender der Deutschen Gesellschaft für Care und Case Management DGCC [6], vertritt Löcherbach zugleich eine Fachdisziplin, die sich dafür berufen fühlt, die mangelnde Abstimmung an Schnittstellen der Versorgung zu verbessern.

Die DGCC hat das im deutschsprachigen Raum führende Curriculum zur Weiterbildung zum Case Manager aufgelegt (siehe das Interview ab Seite 14). Die Absolventen gelten als Spezialisten, wenn es darum geht, überall dort, wo Menschen von verschiedenen Sparten im Sozialsystem versorgt werden, für eine gute Abstimmung aller Akteure zu sorgen.

Manche Krankenhäuser betreiben mittlerweile Abteilungen für Case Management. Im Gespräch mit der ZfOU stellt Sabine Peer, Leiterin solch eines Case Managements am Klinikum-Lüdenscheid die wesentlichsten Arbeitsbereiche vor (siehe das Interview ab Seite 11). Peer argumentiert auch mit harten Zahlen: die Investition in das Case Management zahle sich nicht nur in besserer Versorgungsqualität für den Patienten aus, sondern auch in Euro und Cent für das Krankenhaus.

Ein Vorbild gibt es auch noch aus einem anderen Versorgungsnetzwerk. Die Unfallversicherungen, es gilt das SGB VII, reklamieren ein praxistaugliches Case Management für sich. Allerdings nennt es sich hier - auch das ist beim ungeschützten Terminus Case Management möglich - RehaManagement.

Man arbeite noch am Behandlungserfolg, wenn andere aufgegeben haben, erklärte selbstbewusst Reinhard Nieper, Geschäftsführer der Berufsgenossenschaftlichen Kliniken im Juni 2016 gegenüber der Ärztezeitung [7]. Die Unfallversicherungen haben ein elementares Interesse daran, dass der Patient nach dem Arbeitsunfall wieder in den Beruf zurückkehrt. Dafür arbeitet ein Reha-Manager, angestellt bei der Versicherung, der genau mit dem Patienten austariert, mit welcher Reha und welchen Unterstützungsmaßnahmen er wieder in den Beruf kommt. Notfalls auch über den Weg einer Umschulung. 97\% der Patienten, welche die Reha-Leistungen durchlaufen, so Nieper, kehrten wieder in das Berufsleben zurück. Dieses Reha-Management mache den großen Unterschied zur Regelversorgung aus.
Keine Frage, solch ein individuelles Case Management könnten auch jene Patienten brauchen, die Ulrike Michaelis interviewt hatte. „Wenn man sich die Infos nicht mühsam zusammen klaubt, und dann bleibt man eigentlich ja, alleine in der Wohnung hocken“, sagte Frau L., eine alleinstehende Dame in den 30ern, die ihr Bein verloren hat, zu der Wissenschaftlerin.

Liebes, in vielem zu Recht hoch gelobtes deutsches Sozialsystem - solche Klagen sollten der Vergangenheit angehören!

Literatur

[1] Michaelis U. „Man müsste nur einen Ansprechpartner haben..." Wie major-beinamputierte Menschen ihre rehabilitative Versorgung erleben. Die Entwicklung einer gegenstandsbezogenen Theorie als Basis einer Optimierung der rehabilitativen Versorgung von Menschen vor, während und nach einer Majoramputation am Bein. Inaugural-Dissertation zur Erlangung des Doktorgrades Dr. rer. hum. biol. der Universität zu Lübeck (Sektion Medizin) (2014). Im Internet: http://www.zhb.uni-luebeck.de/epubs/ ediss1511.pdf; Stand: 26.01.2017

[2] Michaelis U, Gaidys U. Optimierung der rehabilitativen Versorgung von Menschen vor, während und nach Majoramputation - Abschlußbericht Projekt Nr. 154 (2012). Im Internet: http://www.reha-vffr.de/internet/ reha-vffr/vffrport.nsf/ispvwLaunchDoc/ 6D78FA1959B0359CC1257914003A91D8/ \$FILE/Bericht_final_2012_12_21.pdf; Stand: 26.01.2017

[3] Verbesserung der Lebensqualität von älteren Menschen mit chronischen Erkrankungen durch Förderung der Selbstpflegekompetenzen am Beispiel von Amputationen. Im Internet: http://www.lebenmitamputation.de/ projektinformationen/; Stand: 26.01.2017

[4] Im Internet: http://www.lebenmitamputation.de/fuer-caremanager/fortbildung-alscaremanager-amputationsbegleiterin/; Stand: 26.01 .2017

[5] Im Internet: https://www.g-ba.de/institution/ presse/pressemitteilungen/595/; Stand: 26.01.2017

[6] Im Internet: http://www.dgcc.de/; Stand: 26.01.2017

[7] Im Internet: http://www.aerztezeitung.de/ politik_gesellschaft/gesundheitswirtschaft/ article/913041/interview-bg-klinik-chef-wirarbeiten-noch-behandlungserfolg-wennandere-aufgegeben.html; Stand: 26.01.2017

Bernhard Epping 